Lexis Vol. XLV (2) 2021: 955-965

\title{
Jeff Siegel. Second Dialect Acquisition. Cambridge: Cambridge University Press, 2010.277 pp.
}

No es usual en el mundo académico reseñar un libro después de una década de su publicación, como es el caso en que me ocuparé. Sin embargo, nunca es tarde para apuntar a una obra, especialmente cuando, desde mi percepción, no ha recibido mucho eco dentro de los estudios lingüísticos hispánicos escritos en español.

Con el desarrollo que ha tenido el estudio del contacto dialectal en Hispanoamérica, como resultado de los movimientos migratorios en las últimas décadas, tal como apuntaré más adelante, es poco frecuente encontrar referencias a la obra que aquí reseño, escrita en inglés, debido al apogeo de la sociolingüística (y otras disciplinas cercanas) en este campo de estudio. Aunque la metodología que se aplica en los estudios de adquisición de un segundo dialecto (en adelante ASD) es muy similar a la que se emplea en los estudios sociolingüísticos — como se podrá comprobar en la obra de Siegel一, la percepción del cambio lingüístico parte de un fundamento teórico distinto debido a que, desde la sociolingüística, el cambio lingüístico (y la variación) que se estudia en el contacto dialectal se observa como una influencia de la variedad receptora, mientras que, desde los principios de ASD, el cambio lingüístico es observado como una adquisición del hablante. Esto marca una gran diferencia que puede enriquecer aun más nuestro conocimiento sobre las variedades lingüísticas, la relación lengua-hablante, los procesos sociocognitivos que favorecen y determinan la adquisición y el aprendizaje lingüístico, así como la descripción de la participación del hablante 
en el dominio de una nueva variedad lingüística, o algunos de sus rasgos. Paso a continuación al resumen, ponderaciones y comentarios adicionales sobre la obra.

Actualmente, es abundante la cantidad de obras enfocadas en describir y explicar los procesos de adquisición de una segunda lengua (en adelante ASL). Sin embargo, no existía una obra centrada en estudiar los procesos de ASD, como señala Jeff Siegel (2010: 21, 35), la cual abarcara los diferentes aspectos lingüísticos y sociolingüísticos que se desarrollan en estos procesos en contextos formales e informales. ¿Por qué existe una proliferación de obras sobre ASL y no sobre ASD? Esta es una inquietud que no aborda el autor y que debió considerar para explicar el vacío que su obra viene a rellenar. Entre las razones que se podrían exponer, es preciso señalar que los resultados obtenidos en los estudios de ASL permiten desarrollar mejores estrategias que se implementan en la enseñanza de una L2 (Lightbown y Spada 2020: 111). Existe una gran demanda en el aprendizaje de una L2; sin embargo, el aprendizaje de un D2 no cuenta con el mismo prestigio.

Sin duda alguna, Siegel elogia en reiteradas ocasiones los logros alcanzados por los sociolingüistas y dialectólogos, quienes han observado los procesos de ASD en situaciones naturales. Sin embargo, señala que, desde esos campos de la lingüística, los procesos de ASD se han abordado principalmente como procesos de acomodación lingüística, (como se describe en la literatura de la sicología social) y no como procesos de aprendizaje lingüístico (como se describe en los procesos de ASL). Al respecto, el autor no profundiza en las diferencias teóricas que resultan de estudiar los procesos de ASD desde estas disciplinas científicas. Desde mi modo de ver, los procesos de acomodación y los de aprendizaje lingüísticos tienen en común que parten de la actitud del hablante frente a la otra variedad cuando entran en contacto.

Second Dialect Adquisition es una de esas obras que se deben convertir en un punto de referencia del objeto que describen. Por ello, este libro es una contribución a la lingüística aplicada y al estudio de ASL. El lector especializado encontrará en sus páginas 
un marco teórico para el estudio de los procesos de ASD, así como distintas metodologías que podrá aplicar en sus propias investigaciones, bien si observa contextos educacionales (o formales), bien contextos educacionales (o informales). A pesar de ello, el autor advierte que, como su libro está enfocado para una audiencia de expertos y no tan expertos en la lengua, no trata algunos temas con la profundidad que algunos lectores esperarían.

¿Qué implica la adquisición de un segundo dialecto (en adelante D2)? ¿En qué se diferencia este aprendizaje del proceso de adquisición de una segunda lengua (en adelante L2)? ¿Son absolutos los criterios que definen un dialecto desde la sociolingüística? ¿Es más fácil adquirir un D2 que una L2? ¿Cuáles metodologías se han utilizado para estudiar la adquisición de un D2 en contextos naturales y escolares? ¿Qué resultados se han obtenido? ¿Cuáles factores sociales influyen en la adquisición de un D2? Estas son algunas de las preguntas que Siegel estudia en este libro enfocado en la ASD.

El autor cuestiona la suposición extendida entre los hablantes de que es más fácil la ASD que la ASL. Sostiene que en dicha suposición existen algunos asuntos que necesitan ser analizados. Al respecto, cita su propia experiencia, e indica que, como hablante de varias lenguas y dialectos, le resulta más difícil realizar un cambio de dialecto que un cambio de lengua (3). Ahora bien, la experiencia del autor, tomada como argumento para sustentar su hipótesis, no es del todo comparable, a mi entender, porque la experiencia citada trata sobre el cambio de una L2 o D2, mientras que la suposición de hablantes se enfoca en la adquisición. Así, el cambio entre lenguas o dialectos supone la adquisición previa de una L2 o D2. No obstante, más adelante presentará argumentos que sustentan la idea de que es más fácil adquirir una L2 que un D2 (capítulo 3 y, específicamente, el 6).

En el primer capítulo, titulado Introduction, Siegel centra su atención en el aprendizaje de un nuevo dialecto y cómo se diferencia del aprendizaje de una nueva lengua. Para ello, trata, con no poco rigor, las diferencias entre lengua y dialecto, argumentando que los criterios de inteligibilidad y el geográfico, aunque son generalmente 
aceptados, no responden a la totalidad de variedades lingüísticas (2). Considerando esto, Siegel adopta un concepto más amplio de dialecto: asume el que se usa en la sociolingüística, según la cual los dialectos son "varieties of the same language that differ from each other in vocabulary, pronunciation and grammar, and that are associated with particular geographic regions or social groups", pero asume también como dialecto a aquellas lenguas, como el danés y el noruego, que "are considered separate languages, although they are mutually intelligeble” (15). Luego describe, a partir de ejemplos tomados principalmente del inglés americano y el australiano (el cual es más cercano al inglés británico), algunas diferencias léxicas, gramaticales, de pronunciación y pragmáticas (aunque las últimas no han sido estudiadas con amplitud en su libro).

En los estudios sobre ASL, el campo de investigación suele dividirse generalmente en contextos naturales y contextos escolares (19). Teniendo en cuenta esta clasificación, el autor dedica los capítulos del 2 al 6 a presentar y analizar distintos estudios de adquisición de un segundo dialecto en un contexto naturalista, mientras que los capítulos del 7 al 9 se muestran estudios en contextos escolares. Algo de lo que carece esta obra es que se limita a resumir solo estudios escritos en inglés y no presenta investigaciones entre contactos hispánicos. Esto abre la puerta a que se redacte otra obra en la que se expongan también los logros alcanzados en los estudios de adquisición de un D2 en contextos hispánicos. Cabe señalar, sin embargo, que los estudios en inglés citados en su libro constituyen una base sólida, objetiva y suficiente para justificar sus argumentos.

En el capítulo 2, "Attainment in naturalistic SDA", se presentan diecisiete estudios sobre los grados de adquisición de un D2 en distintos contactos dialectales con el inglés: el japonés, el brasileño, el noruego, el suizo y el alemán. También presenta, después, tres estudios sobre cambio dialectal (dialect shift) con los detalles metodológicos apropiados. Al final del capítulo, el autor apunta que, como media general entre todos los estudios, solo el $13 \%$ de los sujetos se aproximó a un uso similar nativo de algunas de las variantes del D2 (51). 
En el 3, "Adquiring a second dialect", el autor explica algunas maneras de cómo se adquiere un D2 y la necesidad de representación en el cerebro. Para ello, aplica los conceptos de imitación, reemplazo (replacive o substractive) y adición (aditive), los cuales resultan enriquecedores en el estudio de ASD, ya que permiten describir el grado de adquisición dialectal de los hablantes. Sostiene que la habilidad de imitar un D2 no es evidencia necesaria de una adquisición, aunque reconoce que, basado en Lightbown y Spada (2006: 184), esta estrategia es eficaz en el desarrollo de la entonación y la pronunciación. El concepto de reemplazo refiere a la pérdida del D1 o al cambio estructural en una variable que se puede desarrollar en la adquisición del D2. Finalmente, el término adición alude al dominio del hablante del D1 y el D2, lo que suele denominarse bidialectalismo. Los ejemplos presentados por el autor en este capítulo se centran solo en diferencias fonéticas entre los dialectos. No se presentan, sin embargo, estudios relacionados a variantes léxicas, gramaticales, pragmáticas o culturales entre los dialectos en contacto.

En los capítulos 4 y 5, Siegel explica por qué existen distintos grados de adquisición dialectal en los estudios resumidos en el capítulo 2, los cuales constituyen principalmente el fundamento de sus conclusiones a lo largo de todo el libro. En el 4, "Differential attainment: age effects and linguistic factors", nos muestra que la edad de adquisición fue el factor más determinante en la adquisición de un D2 de su muestra, aunque esta variable social está vinculada, primero, al nivel de la variable lingüística (esto es, fonética, fonológica, morfológica, sintáctica o léxica); y, segundo, a la complejidad de la regla, entendiéndose por regla al conocimiento explícito o al procedimiento computacional implícito que necesita adquirir un aprendiz de un D2 en el proceso de adquisición dialectal.

En "Additional individual and linguistic factors", capítulo 5, continúa presentando otros factores individuales y lingüísticos influyentes en la adquisición de un D2. El tiempo de estancia (lenght of residence), la identidad social, el sexo, la interacción e integración social, la motivación y actitudes sociales, y la ocupación consti- 
tuyen los demás factores individuales o sociales que desarrolla en este capítulo. Pese a que el autor señala que existen pocos estudios sobre actitudes lingüísticas hacia un D2 (117), esta afirmación quizá solo se aplique a la literatura anglosajona consultada para escribir su obra. En la literatura hispánica presente antes de la publicación de su libro, ya se habían realizado numerosos estudios sobre actitudes lingüísticas hacia D2 (Alvar 1983, Martín Butragueño 1993, Almeida Toribio 2000, Rinken y Pérez Yruela 2007, Sancho Pascual 2010, por citar algunos). Los factores lingüísticos que resalta en los siguientes apartados de este capítulo han tenido menos cabida, a mi entender, en la literatura hispánica especializada, por lo cual constituyen unos recursos por explotar que, sin lugar a duda, ayudarán a enriquecer y a explicar algunos desarrollos en la adquisición de un D2 (y el contacto dialectal). La prominencia de la variable (salience) refiere a la característica de ser fácilmente perceptible, prominente o visible (p. 134); la previsibilidad (predictability) consiste en el grado por el cual el aprendiz puede predecir una forma del D2 a partir de la base del D1 (p. 128); y la comprensibilidad (comprebensibility), la cual parte de la hipótesis de que los aprendices utilizan la variante del D2 cuando el uso de la variante del D1 impide la comprensión entre los hablantes del D2 (130).

La suposición de que es más fácil adquirir un D2 que una L2 citada en el capítulo 1 es puesta en cuestionamiento en "The difficulty of SDA" (capítulo 6). La impresión del autor de que le era más fácil cambiar a una L2 que a un D2 es corroborada por algunos investigadores quienes sostienen que el bidialectalismo es más difícil de adquirir que el bilingüismo (Wolfram y Schilling-Estes 1998: 287). Siegel no argumenta esta afirmación de forma apropiada, lo cual constituye una limitación. Resulta difícil para el lector asumir que es más fácil cambiar a una L2 que a un D2. Sí anota, sin embargo, que las pequeñas diferencias lingüísticas, sociales y psicológicas entre dialectos dificultan la adquisición de un D2, ya que no siempre son percibidas por el aprendiz de un D2.

Los capítulos 7 y 8 presentan el estudio de la adquisición de un $\mathrm{D} 2$ en contextos formales. En "SDA in classroom contexts", como 
se titula el capítulo 7, Siegel cita algunas interferencias vernáculas en aprendices del D2 en contextos donde el D1 no juega ningún rol en el aula. Resume distintos estudios donde el D1 de los aprendices es una variedad regional o étnica, o una variedad coloquial, o un pidgin o un creole del inglés. El habla vernácula de estos hablantes presenta distintas dificultades en el entorno escolar porque, a) tienen que aprender a leer y a escribir en una variedad que no es la que conocen (D2); b) como no son considerados aprendientes de una lengua, no reciben instrucciones formales que le ayuden a dominar el D2; c) pueden frustrarse por las constantes correcciones de los profesores, quienes no les permiten expresarse con libertad en su D1, sino que lo denigran o lo consideran inapropiado para el aula (169). Más adelante, el autor reseña algunos estudios, incluyendo el trabajo pionero de Jenny Cheshire (1982), sobre los conflictos entre dialectos en contextos escolares, que pueden contribuir a desarrollar teorías acerca de cómo un hablante de un D1 adquiere un D2 estándar. Siegel está de acuerdo con la afirmación de que “only before God and linguistics are all language equal” (Mackey 1978: 7). Por ello, propone que las diferencias sociales existentes entre los dialectos son algunos de los tantos obstáculos que deben superarse para fomentar programas de adquisición de D2 en las escuelas.

Una de las razones por las que se reprende, corrige o minimiza el uso de las variantes vernáculas en los entornos escolares es la preservación del D2 estándar. Esta ideología es la que tiene presente el autor en el capítulo 8, "Educational approach for SDA" e intenta demostrar que, en lugar de tener un efecto negativo, el uso del D1 en el contexto educativo tiene un efecto positivo. Existen algunos programas educativos en los que se promueve el aprendizaje del D1. En dichos programas, promovidos principalmente para un público adulto, los participantes desean aprender características de otros dialectos por motivos profesionales de rodaje (actores). En otros, sin embargo, se promueve la reducción del acento del D1 por motivos de negocios y trabajo, como sucede en la industria de los centros de llamadas en la India o en Filipinas (195). Asimismo, los resultados obtenidos en contextos escolares donde se partía del 
D1 vernáculo de los estudiantes y luego se comparaban esos usos en el D2 del inglés estándar condujeron a la obtención de mejores puntuaciones en las pruebas de medición de la escritura, la lectura y las habilidades orales, además del aumento de la participación y la motivación de los alumnos (218).

Finalmente, en el capítulo 9, titulado "Explaining the results and taking further steps”, Siegel realiza un acercamiento más profundo respecto a las razones que justifican los resultados positivos y negativos obtenidos en las investigaciones citadas. Reconoce la labor realizada por los sociolingüistas en el estudio de la adquisición dialectal en contextos naturales, a la vez que resalta, una vez más, la necesidad de que se realicen más investigaciones en contextos escolares, como los realizados por Fairclough (2005) y Calchar (2004, 2005).

Esta obra constituye un marco teórico introductorio para el estudio de la ASD, a la vez que expone una disciplina de la ASL que ha recibido poca atención por los investigadores, aun a diez años de su publicación. El aumento de las migraciones internacionales en Hispanoamérica en las últimas tres décadas constituye un campo de investigación lingüística que podría ser abordado desde la perspectiva de la ASD. Aunque estos contextos lingüísticos han sido aprovechados desde la sociolingüística, la dialectología, la lingüística cognitiva, la lingüística de la migración, etc., (si bien no todos con la misma amplitud), los principios que se señalan desde la disciplina de la ASD aportarían otro matiz al estudio de la variedad lingüística en los entornos naturales y especialmente en los escolares.

Finalmente, esta obra ofrece herramientas para abordar los problemas de desestimación del D1 en muchos contextos escolares con mayor presencia de inmigrantes, como los que se han registrado en escuelas de Madrid, donde existen actitudes negativas hacia los dialectos inmigrantes y se reprimen sus usos (Martín Rojo y otros 2003). 
Sirva esta reseña como eco del llamado del autor a desarrollar esta línea de investigación que ya tiene algunos frutos (Varona, Ruiz Peña y Sierra 2018; Gómez 2020).

\author{
Manuel Peralta Céspedes \\ https://orcid.org/0000-0002-9712-8558 \\ Universidad de Murcia \\ manuel.peralta1@um.es
}

\title{
REFERENCIAS BIBLIOGRÁFICAS
}

Almeida Toribio, Jacqueline

2000 "Nosotros somos dominicanos: Language and Self-definition among Dominicans". En Research on Spanish in the United States: Linguistic Issues and Challenges. Ed., Ana Roca. Somerville: Cascadilla Press, 252-270.

Alvar, Manuel

1983 “Español de Santo Domingo y español de España”. Lingüística Española Actual (LEA). V, 225-239.

Calchar, Arlene

2004 "The construction of Creole-speaking students' linguistic profile and contradictions in ESL literacy programs". TESOL Quarterly. 38, 153-165.

Calchar, Arlene

2005 "Creole-English speakers' treatment of tense-aspect morphology in interlanguage written discourse". Language Learning. 55, 275-334.

Cheshire, Jenny

1982 "Dialect features and linguistic conflict in schools". Educational Review. 14, 1, 53-67.

FAirclough, Marta

2005 Spanish and Heritage Language Education in the United States: Struggling with Hypotheticals. Madrid/Frankfurt: Iberoamericana/ Vervuert. 
GómEz, Cenaida

2020 "Aproximación al modelo de adquisición del habla de un segundo dialecto: la producción de la variante [s] por hablantes de tres variedades del español colombiano en contacto en Ciudad Bolívar, Bogotá". Tesis doctoral. Consulta: 20 de febrero de 2021. <https://ir.lib.uwo.ca/etd/6984>.

Lightbown, Patsy y Nina Spada

2006 How Languages are Learned. 3ra edición. Oxford: Oxford University Press.

Lightbown, Patsy y Nina Spada

2020 "Second Language Acquisition". En An Introduction to Applied Linguistics. Eds., Nobert Schmitt y Michael P.H. Rogers. London y New York: Routlegde, 111-127.

Martín Butragueño, Pedro

1993 "Actitudes y creencias lingüísticas en inmigrantes dialectales. El caso de Madrid”. Lingüistica Española Actual (LEA). 15, 265-296.

Martín Rojo, Luisa, María Ángeles Rodríguez, Inmaculada Sierra Rodrigo, Aitana Gari Pérez, Esther Alcalá Rueda y Laura Majares 2003 ¿Asimilar o integrar?: dilemas ante el multilingüismo en las aulas. Madrid: Centro de Investigación y Documentación Educativa. Consulta: 20 de febrero de 2021. $<$ https://sede.educacion.gob.es/publiventa/detalle.action?cod=11236>.

Rinken, Sebastián y Manuel Pérez Yruela

2007 Opiniones y actitudes de la población andaluza ante la migración. Sevilla: Junta de Andalucía.

Sancho Pascual, María

2010 "Actitudes lingüísticas de los inmigrantes ecuatorianos en Madrid”. Lengua y migración. 2, 2, 83-95.

Varona, Daniel, María Esperanza Ruiz-PeÑa, Elkin Sierra y Yasaman RAFAT

2018 "Second dialect and second language imitation of geminates by Colombian Spanish speakers”. En Proceedings of the International Symposium on Monolingual and Bilingual Speech 2017. Ed., Elena Babatsouli. Chania: Institute of Monolingual and Bilingual Speech, 99-106. 
Wolfram, Walt y Natalie Schilling-Estes

1998 American English: Dialects and Variation. Malden, MA: Blackwell.

Recepción: 26/02/2021

Aceptación: 02/07/2021 